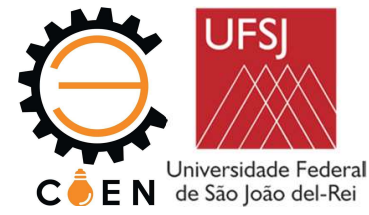

\title{
PROPOSTA DE CÁLCULO DE CURVA QV EM BARRAMENTOS DE CARGA UTILIZANDO REGULADORES DE TENSÃO EM SISTEMAS DE DISTRIBUIÇÃO DE ENERGIA ELÉTRICA
}

\author{
Thauany Oliveira Silva ${ }^{(1)}$ (thauany.oliveira2@cemig.com.br), Renan Souza Moura ${ }^{(2)}$ \\ (renan.moura@ifmg.edu.br), Maristela Costa Rezende Almeida ${ }^{(3)}$ (maaristelalmeida@gmail.com), \\ Mariana Guimarães dos Santos ${ }^{(4)}$ (mariana.santos@ifmg.edu.br) \\ (1) (2) (3) (4) Instituto Federal de Educação, Ciência e Tecnologia de Minas Gerais - IFMG - Formiga - Brasil
}

RESUMO: O interesse mundial pela geração de energia elétrica utilizando fontes renováveis, como a energia fotovoltaica e a energia eólica, despertou, na comunidade acadêmica, a necessidade do conhecimento da modelagem do sistema de distribuição de energia elétrica. Tal interesse incentivou a adaptação de conceitos utilizados em sistemas de geração e transmissão nos sistemas de distribuição, como as curvas QV e PV. A curva $Q V$, ferramenta utilizada em estudos de estabilidade de tensão, permite quantificar o suporte de potência reativa do sistema analisado, determinar o nível crítico de tensão e prever a margem de estabilidade de tensão. É fundamental que um sistema de distribuição de energia elétrica opere de forma adequada, assim, o objetivo desse trabalho foi analisar a variação da margem de estabilidade das curvas QV para barramento de cargas em função do nivel de carregamento do sistema. Outros objetivos secundários foram: avaliar a adaptação da curva QV em sistema de distribuição de energia elétrica, propor uma nova metodologia de cálculo da curva QV utilizando regulador de tensão e realizar ajustes de grandezas elétricas do sistema segundo as normas da ANEEL. A metodologia foi testada no sistema teste "IEEE 4 Node Test Feeder" e em um alimentador de distribuição de energia real da região metropolitana de Belo Horizonte. A curva QV para barramentos de carga foi obtida utilizando reguladores de tensão. Para avaliação da mudança da margem da curva $Q V$, o processo de obtenção da curva QV foi repetido para vários fatores de carga, simulando a transferência de cargas entre alimentadores de distribuição. A metodologia testada permitiu verificar a eficiência da adaptação do conceito da curva $Q V$ em sistemas de distribuição utilizando reguladores de tensão. Também foi verificado o decrescimento da margem da curva $Q V$ na ocorrência de crescimento de carga no sistema. Por fim, o indice retirado de análises da curva QV mostrou um potencial futuro para demonstrar momentos propicios para efetuar correções de fatores de potência.

PALAVRAS-CHAVE: Sistema de Distribuição de Energia, Estabilidade de tensão, Curva QV, Regulador de tensão.

\section{INTRODUÇÃO}

Um sistema elétrico de potência tem como principal objetivo o atendimento às cargas a ele conectadas. De modo geral, esse sistema é formado por geradores, transformadores, linhas de transmissão e alimentadores de distribuição.

Segundo Menezes (2005) a rede de transmissão de energia elétrica tem a finalidade de transportar por meio de cabos aéreos a energia produzida nas usinas para os grandes consumidores e empresas de distribuição. O sistema de distribuição de energia elétrica conecta o sistema de transmissão aos consumidores finais.

Tendo em vista a otimização da eficiência de distribuição de energia, é de extrema importância conhecer os modelos matemáticos das suas linhas. Um modelo matemático descreve o comportamento real de um elemento do sistema através de equações lineares e não lineares. Kersting (2002) utilizou as equações de Carson (1926) para descrever três modelos para linhas de distribuição: modelo completo, que considera valores de impedâncias séries e admitâncias shunts; modelo 

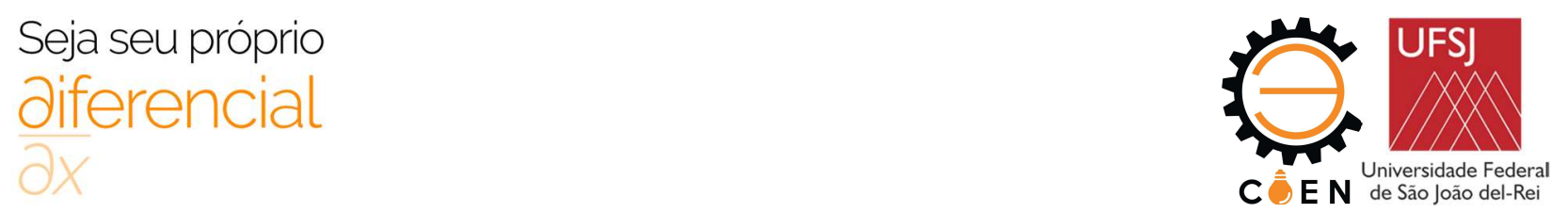

simplificado, que utiliza apenas os valores de impedâncias séries; e modelo aproximado, que é utilizado quando as impedâncias sequenciais das linhas são conhecidas.

Partindo do interesse pela inserção de energias renováveis, a comunidade acadêmica tem feito adaptações de conceitos utilizados em sistemas de transmissão de energia para os sistemas de distribuição de energia elétrica, como por exemplo, a curva QV.

A curva QV tem como principal enfoque a solução de problemas associados à compensação de potência reativa e colapso de tensão, além de possuir facilidade de obtenção mesmo quando o ponto operativo se encontra em pontos operativos instáveis, sendo uma ferramenta utilizada em vários trabalhos como o de Moura et al. (2016) e de Guimarães et al. (2011).

Nas próximas seções será apresentada uma metodologia para a construção de curva QV em barramento de carga para sistemas de distribuição utilizando reguladores de tensão. Em seguida, é realizada uma análise da variação da margem de potência reativa da curva QV.

\section{OBJETIVOS}

Esse trabalho tem como objetivos gerais a implementação de um algoritmo computacional para cálculo da curva QV no barramento de carga em um sistema de distribuição de energia e avaliação do impacto do nível de carregamento do sistema na curva QV. Para atingir estes objetivos, foram desenvolvidos os seguintes objetivos específicos:

- Estudo e validação da proposta de cálculo de curva QV para barramento de carga utilizando o regulador de tensão como equipamento controlador da tensão do barramento.

- Impacto do nível de carregamento do sistema na margem de potência reativa da curva QV.

- Avaliação da influência da adequação as normas da ANEEL do fator de potência e do equilíbrio de carga na margem de potência reativa.

\section{REFERENCIAL TÉORICO}

Esta seção tem como objetivo apresentar uma abordagem teórica para um melhor entendimento dos resultados deste artigo.

\subsection{Fluxo de potência em sistemas de distribuição de energia elétrica.}

Segundo Kersting (2002) um sistema de distribuição é dito não linear quando as cargas são especificadas como potências constantes. Neste caso um método iterativo deve ser usado para calcular as tensões e correntes da carga, ou seja, deve ser realizado o cálculo de fluxo de potência.

Na obra de Kersting (2002) é demostrada a técnica iterativa de progressão utilizada nesse trabalho, sendo a mesma composta de duas partes: varredura para frente e varredura para trás. A varredura para frente calcula as tensões a jusante a partir da fonte, enquanto que com a varredura para trás são obtidas as correntes da carga para a fonte, usando as tensões mais recentes calculadas na varredura para frente. Após a primeira varredura para frente e para trás, é possível calcular novos valores de tensões de carga usando as correntes mais recentes. A varredura continua até que o erro entre as tensões da carga entre duas iterações consecutivas seja menor que um valor de tolerância.

A determinação de impedâncias série de linhas aéreas e subterrâneas é um passo crítico na análise dos alimentadores de distribuição. Carson (1926) escreveu um artigo no qual desenvolveu

IX COEN - Congresso de Engenharias da UFSJ Interconexão. 


\section{Seja seu próprio

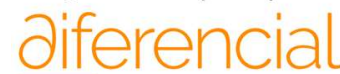

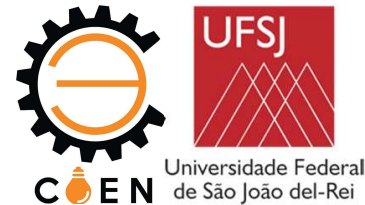

uma técnica onde as impedâncias próprias e mútuas de " $n$ " condutores aéreos e subterrâneos podem ser determinadas. Assumindo que a terra era uma superfície infinita, uniformemente sólida, com uma superfície plana e resistência infinita, ele utilizou o método das imagens de condutores, mostrado na Figura 1, onde cada condutor a uma altura dada tem uma imagem do condutor na mesma distância.

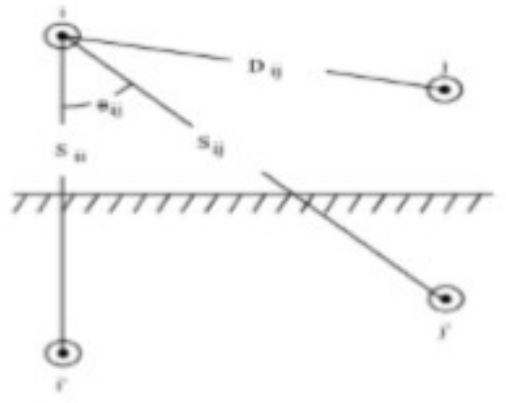

Figura 1. Condutores e imagens.

Fonte: Kersting (2002).

Montemezzo (2016) afirma que o cálculo das admitâncias shunts de linhas, que podem ser calculadas através das equações de Carson (1926), é mais evidente quando se opera em níveis de tensão elevados. Assim, este tipo de efeito pode ser desprezado em sistemas de distribuição e deve ser apenas considerado na modelagem de linhas de transmissão.

Os sistemas de distribuição de energia elétrica exigem uma modelagem trifásica por atender majoritariamente cargas monofásicas e bifásicas, enquanto que a modelagem por diagramas unifilares é suficiente para análises nos sistemas de transmissão.

\subsection{Estabilidade de Tensão}

Segundo Kundur et al. (2004) estabilidade de tensão relaciona-se à capacidade de um sistema de potência de preservar as tensões em regime permanente de todas as barras depois de ser submetido a um distúrbio em uma determinada condição operativa inicial.

Buratti (2016) afirma que a estabilidade de tensão pode ser classificada em duas subcategorias, sendo elas: estabilidade de tensão para um grande distúrbio e estabilidade de tensão para pequeno distúrbio.

A curva QV é uma das ferramentas mais utilizadas nos estudos de estabilidade de tensão e é apresentada a seguir.

\subsection{Curva QV}

Segundo Moura (2016) uma ferramenta útil para análise da sensibilidade de tensão de uma barra, devido à absorção ou injeção de potência reativa, é a curva QV. Em condições de operação normal um aumento da tensão em um barramento é acompanhado de elevação da injeção de potência reativa. Quando ocorre uma diminuição da tensão com a elevação da injeção de potência reativa, esse barramento está operando em uma zona de instabilidade. Esta relação pode ser visualizada de forma fácil utilizando a ferramenta da curva QV. 

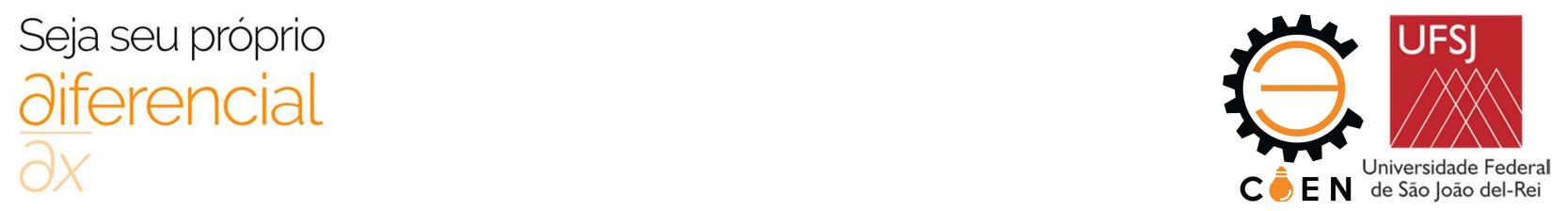

Moura (2016) mostra em sua obra que as regiões estáveis e instáveis de uma curva QV estão representadas, respectivamente, pelos pontos A e B da Figura 2.

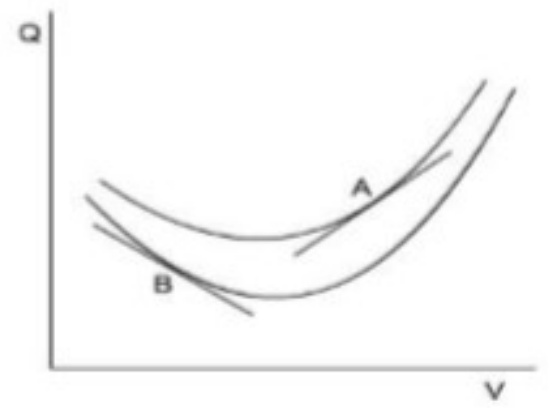

Figura 2. Representação genérica da Curva QV.

Fonte: Moura (2016).

Nos trabalhos de Francisco (2005) e Moura (2016) é demostrado que a margem de potência reativa é definida como a distância do ponto mínimo da curva QV ao eixo horizontal (tensão da barra). A curva QV apresenta margem de potência positiva quando o ponto mínimo estiver acima do eixo horizontal e negativa se tiver abaixo do eixo horizontal. Uma característica relevante em sistemas de transmissão de energia elétrica é o fato que à medida que o sistema se aproxima de um ponto de colapso de tensão, a margem de potência reativa altera-se de negativa para positiva em todos os barramentos.

\subsection{Reguladores de Tensão}

Um regulador de tensão (RT) é basicamente um autotransformador e normalmente possui várias posições de tap, como mostrado na Figura 3. Um RT pode operar tanto na configuração elevador de tensão como na condição de abaixador de tensão.

Segundo Buratti (2016) o que determina se o regulador de tensão é elevador ou abaixador de tensão é a polaridade que a bobina série se encontra. Para isso, há uma chave inversora de polaridade no circuito que é responsável por determinar a operação do regulador com a finalidade de elevar ou diminuir a tensão. Essa comutação é feita com a ajuda de um reator, que tem o objetivo de interromper a circulação de corrente do circuito durante o processo de comutação.

O regulador de tensão foi o equipamento utilizado para controlar a tensão do barramento de carga. Através da variação de tensão do barramento de carga, foram calculadas as respectivas potências reativas com a finalidade de se obter a curva QV.

Neste artigo foram utilizados três reguladores monofásicos conectados em estrela com uma modificação na modelagem de Kersting (2002). A modificação ilustrada na Figura 4 consistiu na exclusão do compensador de queda de linha, que realiza o controle remoto de tensão. Ao traçar uma curva QV para um barramento de carga, o restante do sistema deve ficar em repouso. Sendo assim, um controle remoto de tensão não serve para criar curvas QV. Dessa forma, com a exclusão do controle remoto, o regulador de tensão conseguiu controlar a tensão do seu próprio barramento e foi possível calcular a curva QV para o barramento de carga.

A proposta de utilizar um regulador de tensão para controlar a tensão de um barramento para traçar a curva QV é inovadora. Em trabalhos já realizados sobre a curva QV como o de Lugon et al. 


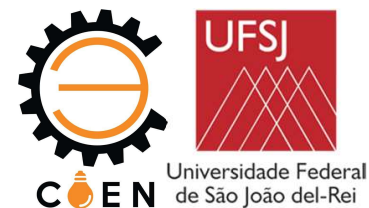

(2009), Pinto (2013) e Reis et al. (2005) é simulado um gerador fictício com limites abertos de geração para obtenção da curva QV do barramento.

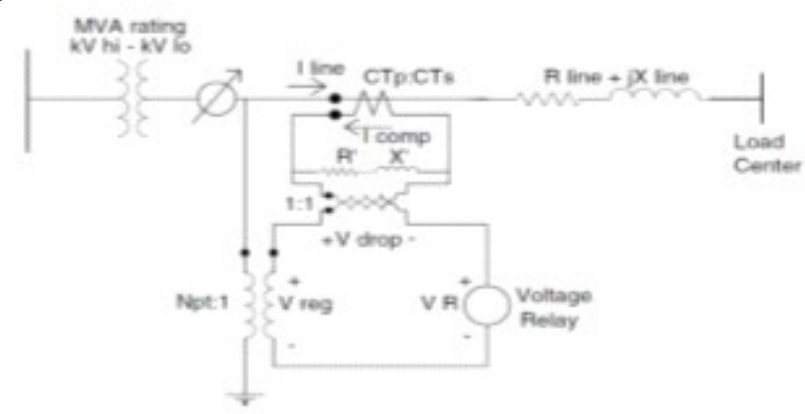

Figura 4. Modificação proposta na modelagem do regulador de tensão.

Fonte: Adaptado de Kersting (2002).

\section{MATERIAIS E MÉTODOS}

\subsection{Sistemas testes}

Como forma de entender a metodologia proposta, foram realizados testes no "IEEE 4 Node Test Feeder", composto por quatro barramentos e normatizado pelo Subcomitê de Análise do Sistema de Distribuição de PSEE, Figura 5.

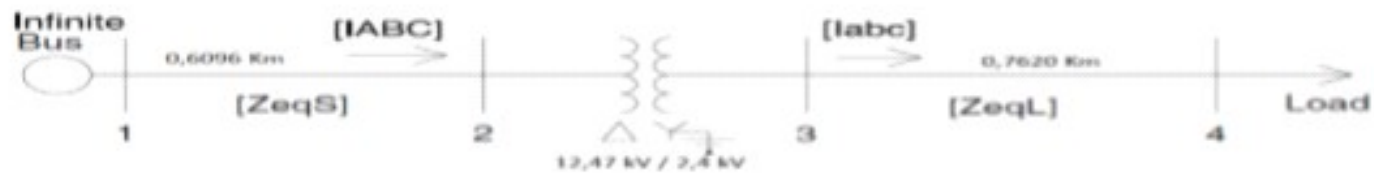

Figura 5. Sistema "IEEE 4 Node Test Feeder".

Fonte: Adaptado de Kersting (2002).

Uma vez compreendida a metodologia, a proposta foi implementada em um alimentador real situado na região metropolitana de Belo Horizonte, cujas características estão indicadas na Figura 6.

\begin{tabular}{|c|c|c|c|c|c|c|c|}
\hline \multicolumn{3}{|l|}{ Dados Geras } & & \multicolumn{2}{|c|}{ Extensठ̄o por Tpo de Plede } & (m) & \\
\hline \multicolumn{2}{|c|}{ Comprimento Tolal } & 12.18 & $\mathrm{Km}$ & Rede Uibana & & Rede Rural & \\
\hline \multicolumn{2}{|c|}{ Comprimento do Tranco } & 10.85 & $\mathrm{Km}$ & Comvencional & 1,11 & Convencional & 0,0 \\
\hline \multicolumn{2}{|c|}{ Comprimento dos Ramais } & 1,32 & $\mathrm{Km}$ & Protegido & 0.08 & Protegida & 0.38 \\
\hline \multicolumn{2}{|c|}{ Número de Tralos } & 5 & & Isclada & 10,31 & Isolada & 0.03 \\
\hline \multicolumn{2}{|l|}{ Demanda Total } & $\longdiv { 1 3 1 7 8 }$ & KVA & Sublenànea & 0,20 & Subterianed & 0.0 \\
\hline \multicolumn{2}{|l|}{ Perdas Totais } & 17.3 & \% & & & & \\
\hline \multicolumn{4}{|c|}{ Número de Equipamentos } & \multicolumn{2}{|c|}{ Caga Instalada (KVA) } & & \\
\hline Reguladoies & 1 & Capacitores & 0 & Distubuida & 150 & Pual & 310 \\
\hline Aelosdares & 2 & Seccion. & 0 & Cancontrada & 3000 & Tolal & 3460 \\
\hline CH Fusivel & 0 & CH Trpolares & 0 & \multicolumn{2}{|c|}{ Número de Clieries } & & \\
\hline CH Unipolere: & 12 & CH TrP. CA & 2 & Socundsrios & 2 & Rurais & 0 \\
\hline Aluto-Troto & 0 & Pt Troo $34.5 k V$ & 0 & Pimbrios & 1 & Tolal & 3 \\
\hline
\end{tabular}

Figura 6. Características do alimentador real do sistema de distribuição de energia elétrica.

Fonte: Autor.

IX COEN - Congresso de Engenharias da UFSJ Interconexão. 


\section{Seja seu próprio

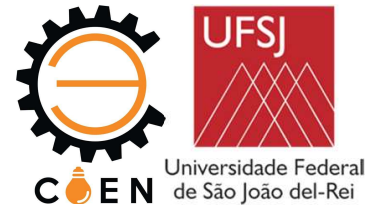

De forma sucinta as etapas utilizadas para o desenvolvimento deste trabalho foram:

1. Cálculo de curvas QV para barramentos de geração e de carga. A curva QV para barramentos de carga é realizada utilizando a modificação proposta no regulador de tensão.

2. Impacto do nível de carregamento na margem de potência de reativa da curva QV de barramentos de carga.

3. Avaliação da influência da adequação a normas da ANEEL do fator de potência e do equilíbrio de fases na margem de potência reativa.

Todas as etapas da metodologia são descritas a seguir.

\subsection{Curva QV para barramento de geração}

A curva QV para barramento de geração é um estudo mais abrangido em trabalhos acadêmicos em comparação a curva QV para barramento de carga. O trabalho de Silva et al. (2016) é um exemplo de trabalho que aborda essa temática.

A curva QV para o barramento de geração foi calculada no sistema teste "IEEE 4 Node Test Feeder" com o intuito de comparar posteriormente com a curva QV para barramento de carga utilizando regulador de tensão.

\subsection{Curva QV em barramentos de carga utilizando regulador de tensão}

\subsubsection{Curva QV em barramentos de carga utilizando regulador de tensão no "IEEE 4 NODE TEST FEEDER"}

O sistema simulado com a inserção do regulador de tensão em cada fase é ilustrado na Figura 7. Foi utilizado um regulador de passo de tensão do tipo B monofásico para cada fase do sistema, conectado em estrela na barra secundária do nó 3 .

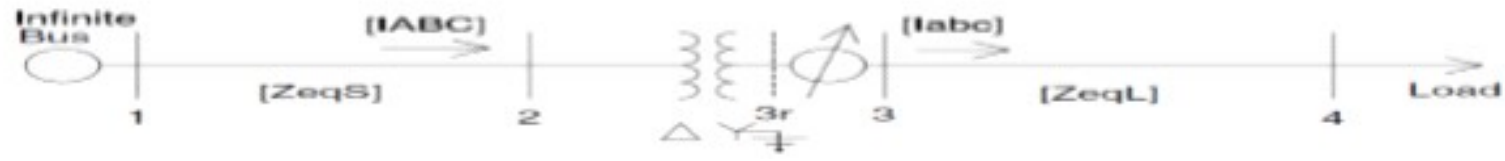

Figura 7. Sistema "IEEE 4 Node Test Feeder" com inserção do regulador de tensão.

Fonte: Adaptado de Kersting (2002).

\subsubsection{Curva QV utilizando regulador de tensão do alimentador de distribuição de energia elétrica real}

Diferentemente do sistema "IEEE 4 Node Test Feeder" que utiliza um regulador de tensão por fase, esse alimentador real possui um regulador de tensão trifásico com ajustes de taps independente para cada fase.

\subsection{Impacto do nível de carregamento do sistema na margem de potência reativa da curva QV.}

Com o objetivo de simular o impacto na margem da curva QV em barramento de carga quando ocorre à transferência total ou parcial de um bloco de cargas entre alimentadores, foi variado o nível 

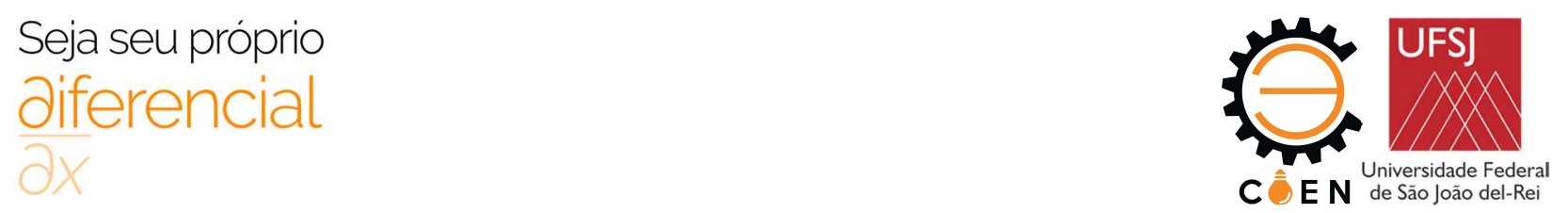

de carregamento do sistema teste através da mudança das potências aparentes consumidas de cada fase.

\subsubsection{Impacto do nível de carregamento no sistema "IEEE 4 NODE TEST FEEDER"}

Para realização dos testes nesse sistema algumas restrições foram consideradas:

- Segundo a norma CEMIG ND-1.1 (2008) o carregamento máximo de cada tronco interligável deve ser em condições normais de operação igual a $60 \%$ da capacidade nominal do condutor predominante ou do ramo de interligação, respeitando-se a necessidade de transferência. $\mathrm{O}$ restante dos $40 \%$ da capacidade nominal do condutor servirá de reserva para o atendimento às ocorrências no sistema elétrico. Dessa forma a simulação de transferência de carga do sistema foi aumentada até no máximo $900 \mathrm{~A}$.

- A potência aparente máxima que o sistema pode fornecer é $2000 \mathrm{kVA}$ por fase.

O aumento do nível de carregamento do sistema foi realizado multiplicando as potências aparentes nominais de cada fase por um fator de carregamento (FC). As Equações 1, 2 e 3 mostram como o nível de carregamento do sistema foi variado na rotina computacional do fluxo de potência. $\mathrm{O}$ valor FC apresentou uma faixa de valores entre 0,8 e 1,2 respeitando as restrições. Nos testes realizados quando o fator de carregamento é menor que 1 , isto significa que houve a simulação de uma falta na fonte de alimentação principal do sistema teste e transferência parcial do bloco de carga. Por outro lado, quando o fator de carregamento é maior que 1, o sistema teste absorve a carga parcial de outro alimentador interligado.

$$
\begin{array}{rrr}
S a=F C \times 750 \mathrm{kVA} & \text { Fator de potência }=0,85 \text { indutivo } \\
S b=F C \times 1000 \mathrm{kVA} & \text { Fator de potência }=0,90 \text { indutivo } \\
S b=F C \times 1000 \mathrm{kVA} & \text { Fator de potência }=0,90 \text { indutivo }
\end{array}
$$

\subsubsection{Impacto do nível de carregamento no alimentador de distribuição real}

Para realização dos testes nesse sistema algumas restrições foram consideradas: • O condutor de fase do sistema teste suporta uma corrente de até 270 A. • A potência aparente máxima que o alimentador do sistema pode fornecer é de 7 MVA.

O aumento do nível de carregamento do sistema foi realizado da mesma forma descrita na seção 4.4.1.

\subsection{Avaliação da influência da adequação a normas da ANEEL do fator de potência e do equilíbrio de fases na margem de potência reativa.}

Foram avaliados dois pontos principais para a adequação aos requisitos normatizados pela ANEEL para o bom funcionamento do sistema de distribuição, o fator de potência e o equilíbrio do carregamento das fases.

A adequação das normas da ANEEL foi realizada apenas no sistema teste "IEEE 4 Node Test Feeder", que possui um desequilíbrio de corrente e fator de potência abaixo do regulamentado. O

IX COEN - Congresso de Engenharias da UFSJ Interconexão. 

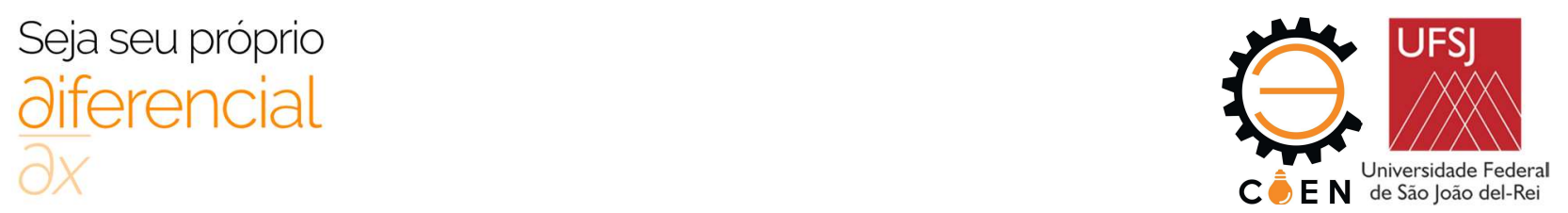

alimentador real por ter sua carga predominante em um consumidor primário trifásico, não possui desequilíbrio acima do recomendado e o fator de potência está dentro do normatizado.

Segundo a ANEEL - Prodist Módulo 8 (2018) uma unidade consumidora ou conexão entre distribuidoras com tensão inferior a $230 \mathrm{kV}$, o fator de potência no ponto de conexão deve estar compreendido entre 0,92 (noventa e dois centésimos) e 1,00 (um) indutivo ou 1,00 (um) e 0,92 (noventa e dois centésimos) capacitivo, de acordo com regulamentação vigente.

Segundo a norma CEMIG ND-1.1 (2008) o desequilíbrio máximo de corrente recomendado em sistemas de distribuição é de $20 \%$, a ser calculado através da Equação 4.

$$
\operatorname{Desequilibrio}(\%)=\frac{3 \times \sqrt{(I a 2+I b 2+I c 2)-[(I a \times I b)+(I a \times I c)+(I c \times I b)] \times 100}}{I a+I b+I c}
$$

Primeiramente foi calculado o desequilíbrio de correntes do sistema com as características nominais e observado a existência de um desequilíbrio de $41,86 \%$, o que ultrapassa os $20 \%$ recomendado pela norma CEMIG ND-1.1 (2008). Dessa forma, foi necessário realizar o equilíbrio do carregamento do sistema, a fim de enquadrar no percentual normatizado. O equilíbrio de fase foi realizado transferindo carga da fase " $\mathrm{C}$ " para a fase " $\mathrm{A}$ " e as respectivas potências após o equilíbrio foram: $S a=950 \mathrm{kVA}, S b=1000 \mathrm{kVA}, S c=1030 \mathrm{kVA}$. O desequilíbrio percentual após a transferência parcial de carga foi $7,05 \%$.

Após o equilíbrio de fases e considerando as novas potências de cada fase, foi realizado o cálculo dos bancos de capacitores necessário para aumentar o fator de potência da fase " $\mathrm{A}$ " de 0,85 indutivo para 0,92 indutivo e da fase " $B$ " de 0,9 indutivo para 0,92 indutivo. A fase " $C$ " não necessita de correção, pois já possui um fator de potência de 0,95 . As potências reativas dos bancos de capacitores da fase "A" e "B" são, respectivamente, 156,45 kVAR e 52,49 kVAR.

Foi traçada a curva QV antes e após a correção do fator de potência e a realização do equilíbrio de corrente, considerando o carregamento nominal do sistema. Assim, foi possível estudar o comportamento da margem de potência reativa da curva QV quando opera dentro e fora dos padrões normatizados.

\section{RESULTADOS}

\subsection{Comparação entre a curva QV para barramento de geração e de carga no sistema "IEEE 4 NODE TEST FEEDER"}

As Figuras 8 (a), (b) e (c) e Tabela 1 mostram a comparação entre as curvas QV para barramento de carga e de geração.

Tabela 1. Comparação entre as margens de potência reativa do barramento de carga e do barramento de geração.

\begin{tabular}{llll}
\hline \multicolumn{4}{c}{ Margens de potência reativa } \\
\hline Barramento de geração & & Barramento de carga & \\
Fase A & $384,257 \times 10^{3}$ VAR & Fase A & $395,145 \times 10^{3}$ VAR \\
Fase B & $438,707 \times 10^{3}$ VAR & Fase B & $436,060 \times 10^{3}$ VAR \\
Fase C & $627,625 \times 10^{3}$ VAR & Fase C & $384,401 \times 10^{3}$ VAR \\
\hline
\end{tabular}

Fonte: Autor.

IX COEN - Congresso de Engenharias da UFSJ Interconexão. 

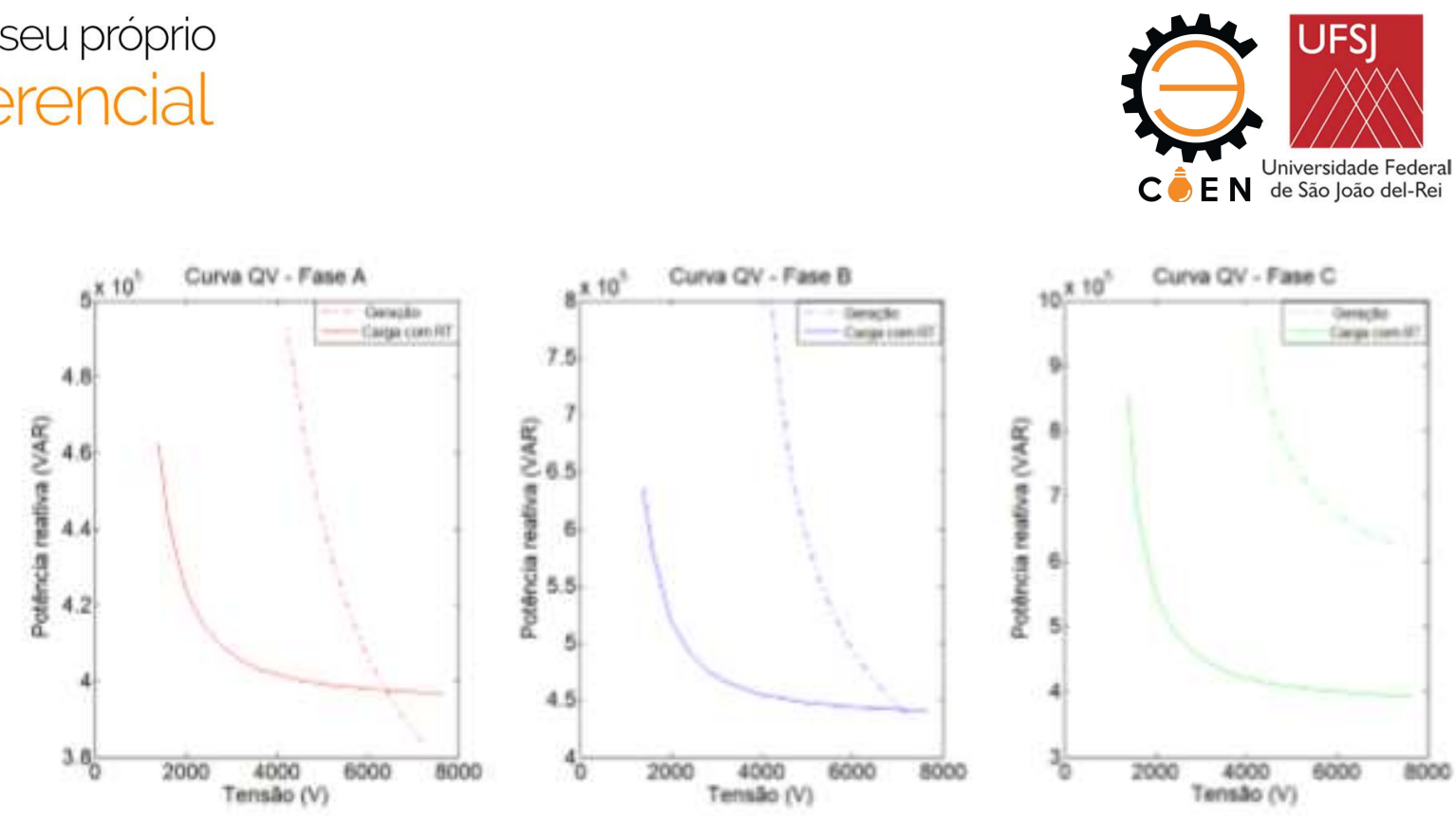

Figura 8. Comparação das curvas QV.
(a) Fase A
(b) Fase B
(c) Fase C

Fonte: Autor.

As margens de potência reativa para barramento de geração e de carga são próximas, pois no sistema "IEEE 4 Node Test Feeder" existem somente impedâncias de pequena magnitude entre estes barramentos. Assim sendo, a metodologia proposta utilizando regulador de tensão se mostrou eficiente.

\subsection{Curva QV utilizando regulador de tensão do alimentador de distribuição de energia elétrica real}

As curvas QV no alimentador real são apresentadas nas Figuras 9 (a), (b) e (c) e as respectivas margens de potência reativa estão indicadas na Tabela 2 .

Tabela 2. Margem de carga da curva QV para o sistema real.

\begin{tabular}{lc}
\hline \multicolumn{2}{c}{ Margens de potência reativa } \\
\hline Fase A & $1334,587 \times 10^{3}$ VAR \\
Fase B & $1247,198 \times 10^{3}$ VAR \\
Fase C & $1380,812 \times 10^{3}$ VAR \\
\hline
\end{tabular}

Fonte: Autor.

IX COEN - Congresso de Engenharias da UFSJ Interconexão. 

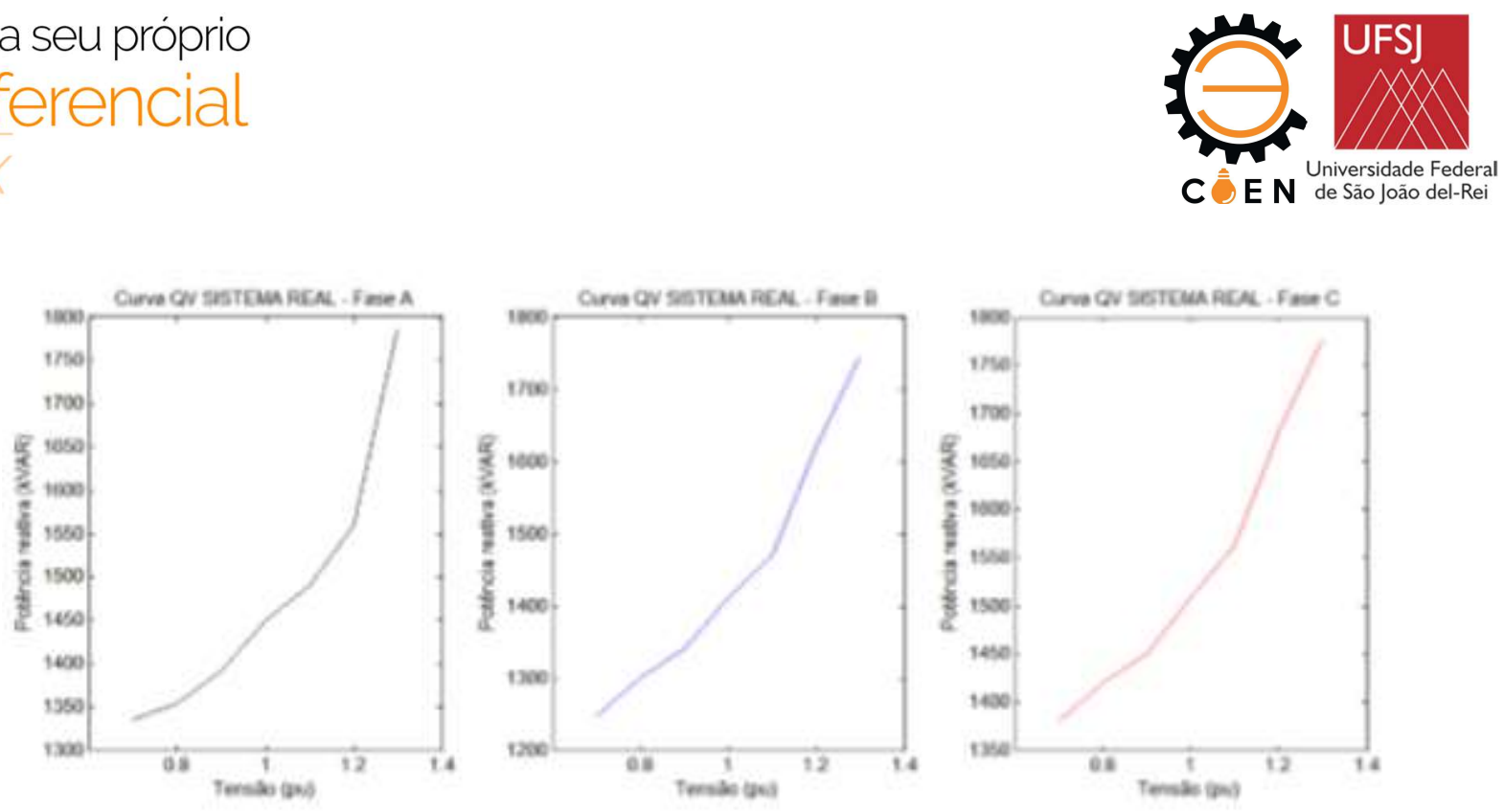

Figura 9. Curva QV do alimentador real.
(a) Fase A
(b) Fase B
(c) Fase C

Fonte: Autor.

O carregamento das fases possui um desequilíbrio de apenas 3,85\% e fatores de potência acima de 0,92 , pelo fato do consumidor predominante do alimentador ser um cliente primário, trifásico, equilibrado e o alimentador possuir apenas mais dois consumidores secundários bifásicos no seu tronco. Todos os pontos operativos estão situados na região de estabilidade de tensão, onde a potência reativa aumenta com o aumento da tensão do barramento.

\subsection{Impacto do nível de carregamento do sistema na margem de potência reativa da curva QV.}

\subsubsection{Impacto do nível de carregamento no sistema "IEEE 4 NODE TEST FEEDER"}

Ao se considerar as restrições operacionais do sistema para calcular a curva QV, observa-se que, com o aumento do fator de carga, a margem de potência reativa mostrada na curva QV das Figuras 10 (a), (b) e (c) diminui. Isto é esperado, pois este aumento de carregamento faz com que os equipamentos e fontes do sistema fiquem mais próximos dos seus limites operativos. Os valores das margens obtidas estão indicados na Tabela 3.

Tabela 3. Margens de potência reativas para os diferentes níveis de carregamento testados

\begin{tabular}{lccc}
\hline Carregamento & Margem da Fase A & Margem da Fase B & Margem da Fase C \\
\hline $\mathrm{FC}=0,8$ & $418,673 \times 10^{3} \mathrm{VAR}$ & $460,258 \times 10^{3} \mathrm{VAR}$ & $407,659 \times 10^{3} \mathrm{VAR}$ \\
$\mathrm{FC}=0,9$ & $404,353 \times 10^{3} \mathrm{VAR}$ & $444,722 \times 10^{3} \mathrm{VAR}$ & $395,642 \times 10^{3} \mathrm{VAR}$ \\
$\mathrm{FC}=1$ & $395,145 \times 10^{3} \mathrm{VAR}$ & $436,060 \times 10^{3} \mathrm{VAR}$ & $384,401 \times 10^{3} \mathrm{VAR}$ \\
$\mathrm{FC}=1,1$ & $364,199 \times 10^{3} \mathrm{VAR}$ & $385,796 \times 10^{3} \mathrm{VAR}$ & $338,565 \times 10^{3} \mathrm{VAR}$ \\
$\mathrm{FC}=1,2$ & $314,938 \times 10^{3} \mathrm{VAR}$ & $351,235 \times 10^{3} \mathrm{VAR}$ & $315,685 \times 10^{3} \mathrm{VAR}$ \\
\hline
\end{tabular}

Fonte: Autor.

IX COEN - Congresso de Engenharias da UFSJ Interconexão. 

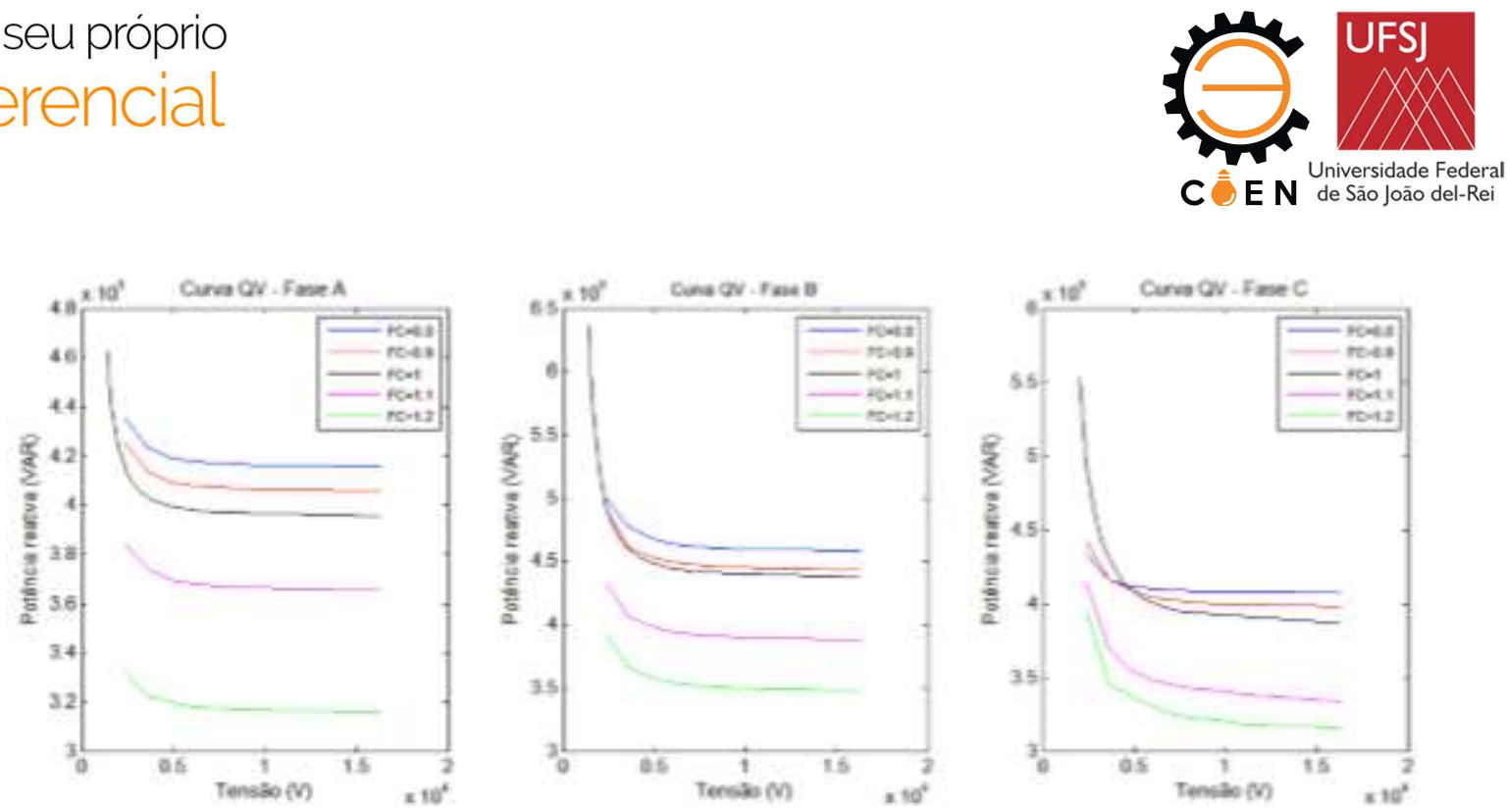

Figura 10. Comparação das curvas QV com variação do nível de carregamento com restrições operacionais.
(a) Fase A
(b) Fase B
(c) Fase C

Fonte: Autor.

\subsubsection{Impacto do nível de carregamento no alimentador de distribuição real.}

Como no sistema teste anterior, ocorre uma diminuição das margens de potência reativa, com o aumento do fator de carga, como pode ser observado nas Figuras 11 (a), (b) e (c), e na Tabela 4.
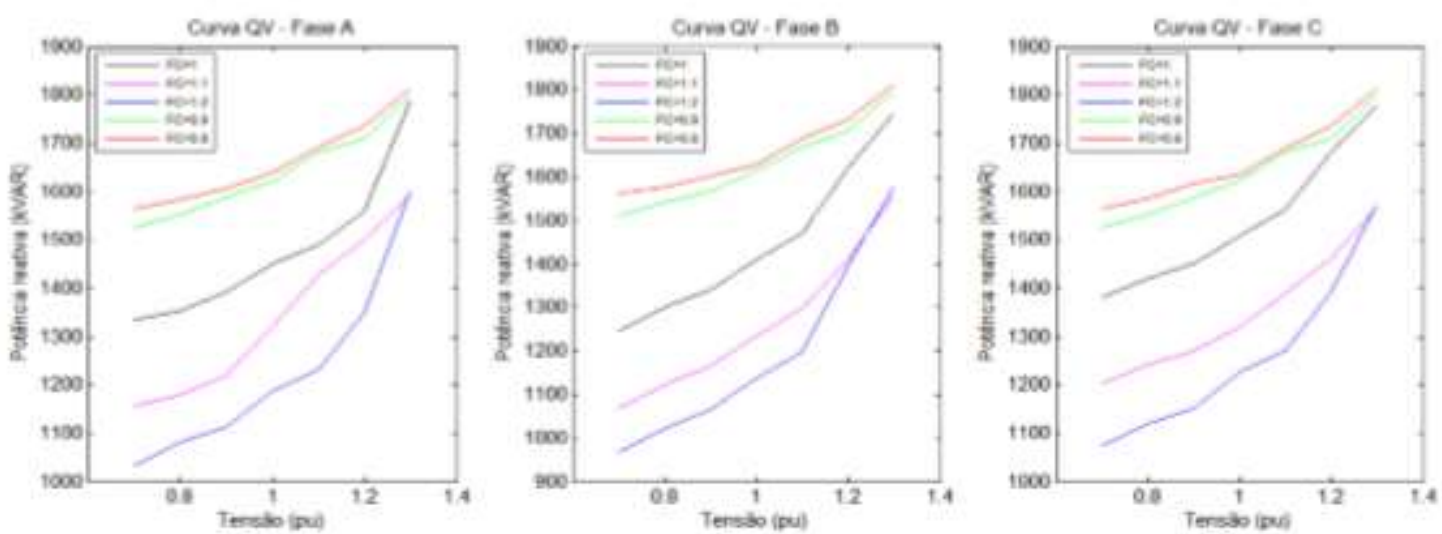

Figura 11. Comparação das curvas QV com variação do nível de carregamento do alimentador real.
(a) Fase A
(b) Fase B
(c) Fase C

Fonte: Autor.

Tabela 4. Margens de potência reativas para os diferentes níveis de carregamento para o alimentador real. 


\begin{tabular}{lccc}
\hline Carregamento & Margem da Fase A & Margem da Fase B & Margem da Fase C \\
\hline $\mathrm{FC}=0,8$ & $1565,408 \times 10^{3}$ VAR & $1561,408 \times 10^{3}$ VAR & $1565,408 \times 10^{3}$ VAR \\
$\mathrm{FC}=0,9$ & $1526,408 \times 10^{3}$ VAR & $1511,408 \times 10^{3}$ VAR & $1529,408 \times 10^{3}$ VAR \\
$\mathrm{FC}=1$ & $1334,587 \times 10^{3}$ VAR & $1247,198 \times 10^{3}$ VAR & $1380,812 \times 10^{3}$ VAR \\
$\mathrm{FC}=1,1$ & $1157,408 \times 10^{3}$ VAR & $1068,608 \times 10^{3}$ VAR & $1201,812 \times 10^{3}$ VAR \\
$\mathrm{FC}=1,2$ & $1031,408 \times 10^{3}$ VAR & $966,10 \times 10^{3}$ VAR & $1073,408 \times 10^{3}$ VAR \\
\hline
\end{tabular}

Fonte: Autor.

\subsection{Avaliação da influência da adequação a normas da ANEEL do fator de potência e do equilíbrio de fases na margem de potência reativa.}

As Figuras 12 (a), (b) e (c) mostram uma comparação entre as curvas QV do sistema teste "IEEE 4 Node Test Feeder" para diferentes níveis de carregamento e para o sistema operando com um equilíbrio de carga, fator de potência acima de 0,92 e carregamento nominal.

Como é possível notar, as curvas QV com as adequações as normas, estão entre as curvas com características nominais e com fator de carregamento de 0,9. Esse fato pode ser explicado porque um sistema com bom fator de potência (acima de 0,92) precisa de menos corrente elétrica para efetuar o mesmo trabalho do que na condição com um baixo fator de potência.

Quando é realizado o ajuste do fator de potência e equilíbrio da carga das fases ocorre um alívio no sistema. Tal fato evidencia a possibilidade de estudar em trabalhos futuros a curva QV como índice para determinar situações onde devem existir correções de fator de potência.
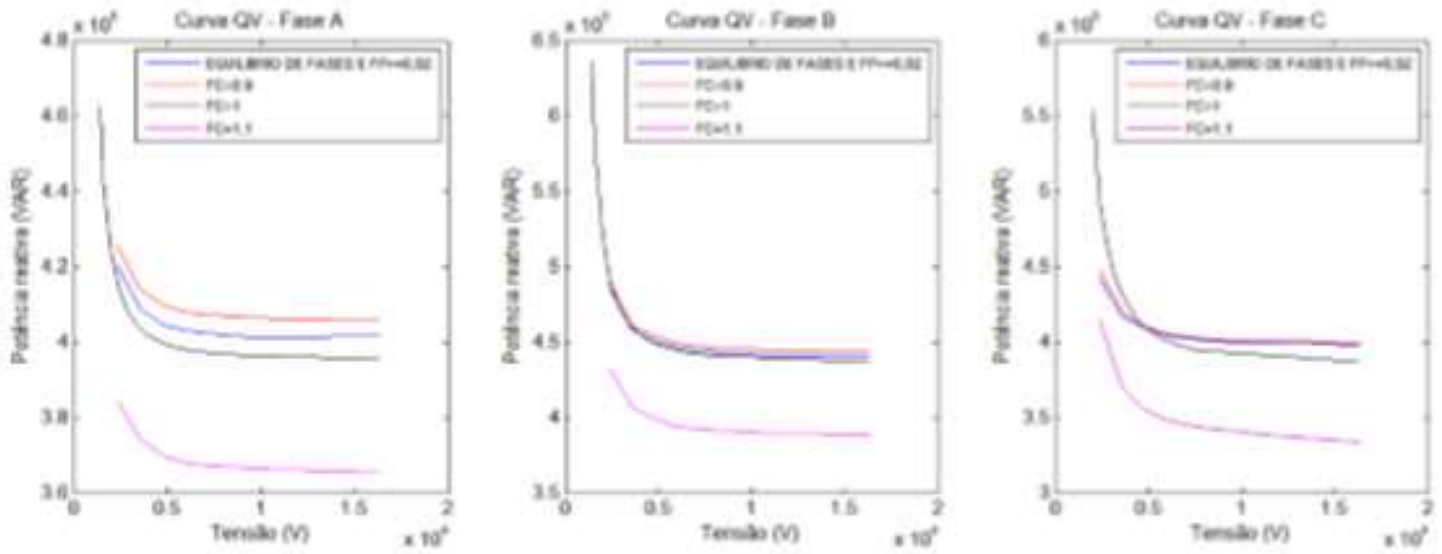

Figura 12. Comparação das curvas QV com variação do nível de carregamento e adequação a norma.
(a) Fase A
(b) Fase B
(c) Fase C

Fonte: Autor.

\section{CONCLUSÕES}

A metodologia proposta de utilização de reguladores de tensão para cálculo da curva QV para barramento de carga se mostrou eficiente, pois a modificação permite o controle local de tensão, algo necessário para traçar curvas QV. 

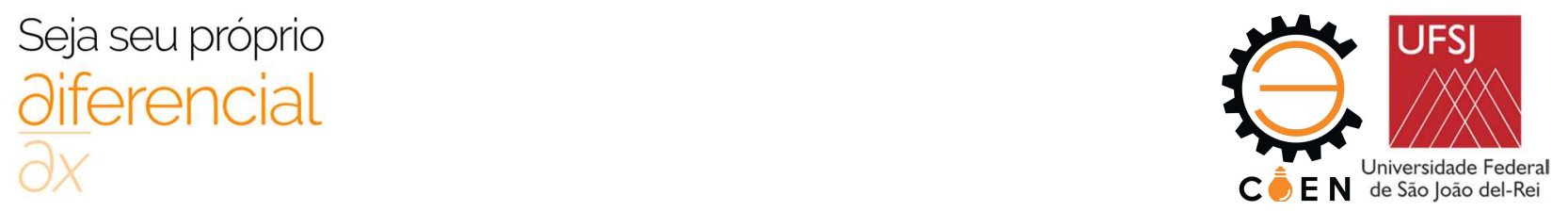

Os resultados nos sistemas testes demonstraram que à medida que um sistema aumenta a sua carga, a sua margem de potência reativa da curva QV diminui. Este resultado está de acordo com a Literatura, pois uma menor margem na curva QV significa uma maior proximidade do ponto de instabilidade de tensão.

A adaptação às normas da ANEEL em relação ao fator de potência e equilíbrio de cargas utilizando a curva QV se mostrou uma alternativa para realizar um alivio de carga no sistema, atenuando a ocorrência de sobrecarga e perda de energia / ineficiência energética.

\section{AGRADECIMENTOS}

A todos os integrantes do Grupo de Pesquisa CNPq, GSE (Grupo de Soluções em Engenharia), pela interação e colaboração no desenvolvimento do presente trabalho.

\section{DIREITOS AUTORAIS}

Os autores são os únicos responsáveis pelo conteúdo das informações contidas neste artigo.

\section{REFERÊNCIAS}

ANEEL. Procedimentos de Distribuição de Energia Elétrica no Sistema Elétrico Nacional PRODIST- Módulo 8. Disponível em: < http://www.aneel.gov.br/modulo-8> . Acesso em 03 junho 2019.

BURATTI, R. P. Reguladores de tensão em redes elétricas com alta penetração de cargas não lineares. Dissertação de Mestrado em Engenharia Elétrica - Faculdade de Engenharia do Campus de Ilha Solteira, 137 p., 2016.

CARSON, J.R. Wave propagation in overhead wires with ground return, Bell System Technical Journal, 5, 539, 1926.

FRANCISCO, G. S. Avaliação de Ferramentas de Análise de Estabilidade de Tensão. Dissertação de Mestrado em Engenharia Elétrica - Universidade Federal de Itajubá, 163 p., 2005.

GUIMARÃES, P. et al. QV and PV curves as a planning tool of analysis, 4th International Conference on Electric Utility Deregulation and Restructuring and Power Technologies (DRPT), Weihai, Shandong, v.4, p. 1601-1606, 2011.

KERSTING, W. H., Distribution System Modeling and Analysis, CRC Press, Second Edition, New York, USA, 2002.

KUNDUR, P. et al. Definition and classification of power system stability. IEEE Transactions on Power Systems, v. 19, n. 3, p. 1387-1401, 2004.

LUGON, V. B. et al. Curva QV E Índice HHI Para Análise de Colapso de Tensão e Monopólio de

Potência Reativa. Belém, $2009 . \quad$ Disponível
em:<http://www.mfap.com.br/pesquisa/arquivos/20090923140708-10.pdf >. Acesso em: 02 maio. 2018.

MENEZES, V. P. Linhas de transmissão de energia elétrica aspectos técnicos, orçamentários e construtivos. Dissertação de Trabalho de Conclusão de Curso em Engenharia Elétrica - Universidade Federal do Rio de Janeiro, 87 p., 2015.

MONTEMEZZO, J. F. Influência dos Parâmetros e Modelos de Linha na Solução do Fluxo de Carga de Sistemas de Distribuição de Energia Elétrica. Dissertação de Mestrado em Sistemas Dinâmicos e Energéticos - Universidade Estadual do Oeste do Paraná, 124 p., 2016.

IX COEN - Congresso de Engenharias da UFSJ Interconexão. 

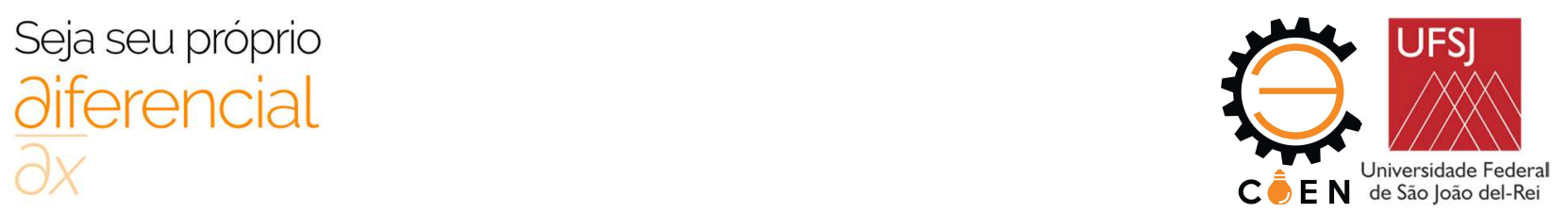

MOURA, R. S. Estudo do efeito do tipo da curva QV em simulações dinâmicas em sistemas elétricos de potência. Dissertação de Pós-Graduação em Engenharia Elétrica - Universidade Federal de Itajubá, 87 p., 2016.

MOURA, R. S. et al. Effects of QV curves in the dynamic behaviour of power systems. IET Generation, Transmission\&Distribution, v. 10, n. 12, p. 2861-2870, 2016. Norma CEMIG ND-1.1, Critérios Técnicos para o Planejamento Elétrico da Distribuição de Média Tensão, Belo Horizonte, 2008.

PINTO, S. S. Comparação da Função Energia com Curvas P-V e Q-V na Análise de Estabilidade de Tensão. Dissertação de Mestrado em Engenharia Elétrica - Universidade Federal de Itajubá, 106 p., 2013.

REIS, C. S. M. M. dos et al. Determinação de índices de estabilidade de tensão. Dissertação de Mestrado em Engenharia Elétrica - Universidade do Porto, 90 p., 2005.

SILVA, G. F. et al. Tratamento de Limites de Geração de Potência Reativa no Problema de Fluxo de Potência Utilizando Análise de Sensibilidade na Formulação de Critérios de Backoff. VI Simpósio Brasileiro de Sistemas Elétricos (SBSE), v.6, p. 32-45, 2016.

\title{
PROPOSAL FOR CALCULATION OF QV CURVE IN LOAD BUS USING VOLTAGE REGULATORS IN ELECTRICITY DISTRIBUTION SYSTEMS
}

\author{
Thauany Oliveira Silva ${ }^{(1)}$ (thauany.oliveira2@cemig.com.br), Renan Souza Moura ${ }^{(2)}$ \\ (renan.moura@ifmg.edu.br), Maristela Costa Rezende Almeida ${ }^{(3)}$ (maaristelalmeida@gmail.com), \\ Mariana Guimarães dos Santos ${ }^{(4)}$ (mariana.santos@ifmg.edu.br) \\ (1) (2) (3) (4) Instituto Federal de Educação, Ciência e Tecnologia de Minas Gerais - IFMG - Formiga - Brasil
}

ABSTRACT: The world's interest in generating electricity using renewable energy sources, such as photovoltaics and wind energy, has awakened in the academic community the need to know the modeling of the electricity distribution system. This interest encouraged the adaptation of concepts used in generation and transmission systems in distribution systems, such as the $Q V$ and $P V$ curves. The $Q V$ curve, a tool used in voltage stability studies, allows quantifying the reactive power support of the analyzed system, determining the critical voltage level and predicting the voltage stability margin. It is fundamental that an electrical system operates properly, so the objective of this work was to analyze the variation of the stability margin of the $Q V$ curves for load buses as a function of the loading level of the system. Other secondary objectives were: to evaluate the adaptation of the $Q V$ curve in the electric energy distribution system, to propose a new methodology for calculating the $Q V$ curve using voltage regulator and to make adjustments of the electrical quantities of the system according to ANEEL standards. The methodology was tested in the test system "IEEE 4 Node Test Feeder" and in a feeder of real energy distribution of the metropolitan region of Belo Horizonte. The QV curve for load rails was obtained using voltage regulators. In order to evaluate the change in the margin of the $Q V$ curve, the process of obtaining the $Q V$ curve was repeated for several load factors, simulating the transfer of loads between distribution feeders. The tested methodology allowed verifying the efficiency of the adaptation of the concept of the $Q V$ curve in distribution systems using voltage regulators. It was also verified the decrease of the margin of the $Q V$ curve in the occurrence of load growth in the system. Finally, the index taken from analyzes of the QV curve showed a potential to demonstrate propitious moments to make corrections of power factors.

PALAVRAS-CHAVE: Power Distribution System, Voltage stability, QV curve, Voltage regulator. 